\title{
Corridoi di connessioni. Rinselvatichire l'ambiente, ripristinare relazioni
}

Hallways of connections. Rewildening the environment, rebuilding relations

\section{Lia Zola}

\section{OpenEdition}

\section{Journals}

\section{Edizione digitale}

URL: https://journals.openedition.org/aam/4629

DOI: $10.4000 /$ aam.4629

ISSN: 2038-3215

Editore

Dipartimento Culture e Società - Università di Palermo

\section{Notizia bibliografica digitale}

Lia Zola, «Corridoi di connessioni. Rinselvatichire l'ambiente, ripristinare relazioni», Archivio antropologico mediterraneo [Online], Anno XXIV, n. 23 (2) | 2021, online dal 31 décembre 2021,

consultato il 08 janvier 2022. URL: http://journals.openedition.org/aam/4629 ; DOI: https://doi.org/ $10.4000 /$ aam.4629

Questo documento è stato generato automaticamente il 8 janvier 2022.

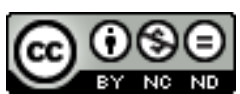

Archivio antropologico mediterraneo è distribuita con Licenza Creative Commons Attribuzione - Non commerciale - Non opere derivate 4.0 Internazionale. 


\section{Corridoi di connessioni. Rinselvatichire l'ambiente, ripristinare relazioni}

Hallways of connections. Rewildening the environment, rebuilding relations

Lia Zola

\section{Le piante, da "sfondo verde" ad attori sociali}

1 Le piante sono rimaste per decenni elementi secondari e passivi agli occhi dell'antropologia: sono state generalmente meno considerate rispetto alle loro controparti non-umane animali, indicate comunemente con termini generici quali habitat, paesaggio o agricoltura o, ancora, ritenute poco più di uno «sfondo verde per l'attività umana» (Rival 2016: 147). Solo di recente sembra che diverse discipline, incluse le scienze sociali, abbiano preso coscienza sia del ruolo benefico che le specie vegetali hanno rivestito, e continuano a farlo, nei confronti degli umani e dei nonumani (Smith et al. 2011; Mancuso 2017), sia del fatto che instaurano relazioni e connessioni con essi.

2 La situazione attuale appare però abbastanza controversa: se da un lato, almeno in Occidente, ci si sta rendendo conto di quanto le piante siano centrali per la sopravvivenza di più specie, dall'altro, quasi in concomitanza con un declino nell'educazione botanica (Kramer, Havens 2015), la conoscenza del mondo vegetale si sta gradualmente perdendo (Wandersee, Schussler 2001). L'urbanizzazione crescente e la mancanza di contatto diretto hanno portato, come sostengono Wandersee e Schussler, ad una «cecità verso le piante» (2001: 4), un'incapacità a distinguere e interpretare concettualmente un mondo botanico che è stato spogliato del suo significato. La "cecità" in questione si riferisce in particolare al fatto che nella vita quotidiana generalmente non si fa caso alle piante e, di conseguenza, saperi e conoscenze relative al loro ciclo di vita stanno scomparendo. Secondo i due autori citati, i motivi per cui non vengono notate e, quindi, non suscitano particolare 
interesse, sono due: le piante non si muovono e hanno tempi lunghi di crescita. Tuttavia, sebbene non si spostino per cercare un compagno, come avviene invece per altre specie non-umane, alcune di loro sono riuscite ad aggirare l'ostacolo attraverso una serie di caratteristiche che permettono l'impollinazione: i fiori. Più attraente è la fioritura, più frequentemente gli insetti pronubi le visitano e, di conseguenza, maggiore è la quantità di semi che gli impollinatori spargono. Questo dimostra che le piante sono in grado di controllare la relazione con gli insetti impollinatori attraverso stimoli visivi che hanno affinato nella loro co-evoluzione (Ibidem; Mancuso 2017). Avere dei tempi lunghi di crescita, inoltre, fa di loro entità che danno una forte impronta al territorio in cui risiedono e che mutano con il tempo, collocandosi in un ambito di interazioni sociali all'interno e oltre l'umano. Le piante, dunque, hanno una «lentezza fallace» (Lewis-Jones 2016: 3) perché sono comunque comprese nella «lotta tra la vita e la morte per avere comunque il diritto di stare in un certo luogo e in un certo momento» (Latour 2014: 15).

3 Gli studi sulla domesticazione hanno mostrato che i destini dell'uomo e delle piante si sono incrociati molte volte, creando legami di mutua dipendenza (Cassidy, Mullin 2007; Rival 2007; Zeder 2006). Se l'attenzione delle scienze sociali è stata per lo più rivolta alle specie domesticate, è oltremodo importante riconoscere che non sono le uniche esistenti e che molte altre hanno comunque intersecato la vita degli umani, proprio come delle linee che ad un certo punto entrano in relazione con altre (Ingold 2015). In altre parole, abbiamo sempre condiviso - e continuiamo a farlo - lo spazio con le piante in una «sopravvivenza collaborativa» che è ben lungi dal collocarle come cornici, o «sfondi verdi» (Tsing 2015: 42).

4 A partire dalla fine degli anni Novanta si è fatto strada un approccio fondamentale nel plasmare e dare corpo ad una serie di contributi che considerano le specie vegetali come attori sociali. Queste riflessioni sono scaturite da una radicale messa in discussione di categorie e dualismi che fino a poco tempo prima sembravano indubbi, tra cui natura e cultura, umani e non-umani. Promossa da autori quali Philippe Descola (1992, 1996, 2000, 2020), Tim Ingold (2000, 2015), Eduardo Viveiros de Castro (1998, 2015), Bruno Latour $(2004,2005,2014)$, Eduardo Kohn (2007, 2013), Donna Haraway $(2008,2016)$, Anna Tsing $(1995,2015)$ tra gli altri, la "svolta ontologica" propone di "passare attraverso» (pass through in originale, Viveiros de Castro et al. 2014: 1) ciò che studiamo, favorendo una destrutturazione di coppie oppositive quali natura e cultura, umano e non-umano, in un'antropologia aperta che integri piante, animali ma anche oggetti e fenomeni atmosferici in un approccio multispecie.

5 L'antropologia si è a lungo occupata di differenze culturali e sociali, cercando di comprendere in che modo le prospettive sul mondo cambiano a seconda dei contesti in cui esse si sviluppano. In questo senso il concetto di cultura, o di società, è sempre stato inteso come qualcosa che tutti possiedono ma il cui contenuto può variare (Strathern 1995). Per chi sostiene la "svolta ontologica" questa preoccupazione per le differenze implica, al contrario, una visione secondo la quale le cose su cui gli individui hanno prospettive differenti sono, in realtà, sempre le stesse. Le persone vedono il mondo in modi diversi, ma il mondo è sempre il mondo. Uno degli esempi maggiormente significativi, a tal riguardo, avviene con il linguaggio: la terra può essere chiamata earth in inglese, terre in francese, ЗЕМЛЯ in russo; ogni espressione porterà con sé uno spettro più o meno ampio di connotazioni simboliche, ma la terra rimarrà sempre la terra. $\mathrm{A}$ partire da un esempio come quello appena riportato, la stessa nozione di differenze 
"sociali" o "culturali" implica il loro esatto opposto, e cioè l "unità" naturale: questa sorta di relativismo culturale in realtà non è abbastanza relativista (Henare et al. 2006, Holbraad 2009) perché relativizza le prospettive sul mondo e quindi universalizza la natura del mondo stesso. Le epistemologie, e cioè le forme del sapere e della comprensione, possono variare, ma esiste solo un'ontologia, e cioè la forma dell'essere o dell'esistere. In buona sostanza, molte visioni del mondo, ma un mondo solo.

Per i sostenitori della "svolta ontologica", al contrario, i mondi, così come le visioni dello stesso, possono variare. Quest'idea implica notevoli sviluppi principalmente per l'antropologia, ma anche per tutte quelle discipline in cui cultura e società, così come altre coppie oppositive, possono essere non solo decostruite ma completamente ribaltate. Lo studio delle relazioni umano non-umano, dunque, dà risalto alle ontologie e alle soggettività; suggerisce inoltre che concentrarsi esclusivamente sulla categoria di umano renda solo sguardi interni, modelli di significato inerenti unicamente alla nostra specie.

\section{Verso una "svolta delle piante"?}

7 Alla luce di queste considerazioni, questo approccio si chiede fino a che punto le specie vegetali siano attori sociali che fanno uso di strategie, che pensano e agiscono. Si chiede anche come le scienze sociali utilizzino le categorie di agency e di persona quando si parla di piante. Ragionare su queste questioni si è dimostrato un mezzo analitico molto efficace (Kohn 2013; Marder 2013): nel contesto del decentramento dell'umano e della migliore comprensione del "più che umano" (Kohn 2007), sembra che le piante possano contribuire in modo decisivo. Se si presta attenzione al loro comportamento, diventa sempre più chiaro che esse «fanno il mondo» (Sheridan 2016: 36), modellando le reciproche esistenze ed esperienze; le loro interazioni e "ontologie vegetali" (Marder 2013) hanno molto da insegnarci non solo su altri modi di vivere e abitare il mondo ma, soprattutto, di cosa significhi essere umani (Ogden et al. 2013).

8 L'etnografia multispecie accoglie queste istanze in una sorta di "svolta delle piante", in cui ciò che emerge è, appunto, la relazione. Eduardo Kohn a riguardo afferma che la foresta pluviale amazzonica è in grado di «pensare come un assemblaggio multispecie» (2013: 83) che comunica attraverso meccanismi di crescita e di decadimento. Invece che focalizzarsi sui runa e sulla loro foresta come un insieme socio-ecologico, Kohn rivela che si tratta invece di un sistema semiotico-ecologico: tutte le forme di vita producono significati e, di conseguenza, possono pensare e apprendere in un'ecologia del sé che oltrepassa i confini dell'umano (Kohn 2013). Sia Kohn, sia altri autori che si sono confrontati con la prospettiva multispecie riguardo alle piante (Berrigan 2014; Hartigan 2015; Archambault 2016), hanno messo in luce il fatto che esse hanno un certo livello di agency e soggettività, dal momento che «le loro vite, le loro esistenze plasmano e sono plasmate da forze politiche, economiche, culturali» (Kirksey, Helmreich 2010: 545). Questa prospettiva è particolarmente utile perché si concentra sull'agency vegetale ma allo stesso tempo su come quest'ultima viene elaborata culturalmente da istituzioni e simboli. Questo trova particolare riscontro nelle affermazioni sia di Bruno Latour sia di Tim Ingold. Il primo sostiene che le entità esistano solo come nodi di sistemi di relazione. Non ci sono oggetti, solo relazioni: persone e piante possiedono caratteristiche solo in virtù delle loro connessioni con altre entità (Latour 2005; Gershon 2010). I non-umani, a tal riguardo, sono per Latour le masse mancanti 
dell'universo sociale e possono esserlo nella misura in cui non costituiscono, come siamo abituati a pensare, una materia inerte a nostra disposizione per l'articolazione delle relazioni ma sono soggetti attivi nei processi di associazione e continua "riassociazione" (Latour 2005) delle realtà sociali. è solo grazie ai non-umani, e alla loro capacità di produrre autonomamente effetti, che tante situazioni sociali prendono una certa forma e riescono a stabilizzarsi in essa nonostante le molte forze divergenti cui sono continuamente sottoposte. Latour a tal proposito ha introdotto il termine "attante" per descrivere qualsiasi essere che abbia l'agency per influenzare altri nodi, altri punti del network.

9 Ingold, nelle sue produzioni più recenti (2015), propone di sostituire il paradigma del network con quello del meshwork, ovvero un tessuto di fasci e linee intrecciate, di vita, crescita e movimento. Secondo Ingold l'essere vivente è una linea mobile, un intreccio di fili e di altre linee, insiemi di nodi che si alterano e si modificano continuamente. Per questo motivo suggerisce di modificare il concetto di abitare "nel" mondo (dwelling, Ingold 2000) con quello di abitare "lungo" il mondo, "con" il mondo (inhabiting): noi viviamo e ci muoviamo non in un ambiente, ma lungo un ambiente, fatto di entità che si spostano, si incontrano, facendo(si) vita a vicenda (Ingold 2015, Perullo 2016). Ritorna dunque, anche se con le dovute differenze tra i due autori, un'idea di relazionalità interspecie che è prima di tutto un'intersezione, una confluenza di molti tipi di interazione.

10 Tutto ciò dimostra che lo studio dei non-umani vegetali rientra a pieno titolo nel dibattito relativo alla multispecie: se da un lato si pone come uno degli sviluppi più interessanti scaturiti dalla "svolta ontologica", dall'altro non si occupa solamente di "guardare attraverso" o "oltre" categorie e dualismi menzionati precedentemente, ma anche di evidenziare le continuità relazionali tra specie diverse e, come si vedrà in seguito, «tra ontologie scientifiche, in particolare quelle che studiano le piante, e le ontologie native» (Cuturi 2020: 230).

11 Un caso particolarmente interessante è rappresentato dalle piante che marcano $\mathrm{i}$ confini. Si tratta di specie diverse, generalmente arbustive spontanee come la cordilina (Cordyline fruticosa), la rosa canina (Rosa Canina), il corniolo (Cornus mas) o il biancospino (Crataegus Levigata) che, come suggerisce il loro nome, vengono utilizzate per circoscrivere certe aree: possono essere utilizzate, ad esempio, per contenere il bestiame, per segnare il limite tra due o più campi coltivati ma anche per circondare il perimetro di una nuova abitazione o dei luoghi di sepoltura. Le piante che marcano $\mathrm{i}$ confini si collocano esattamente alla confluenza tra ambiti diversi e sono parte di un sistema di relazioni più ampio che comprende l'ecologia del paesaggio, la struttura sociale, i significati culturali ma anche le negoziazioni per la terra e i conflitti che questi ultimi veicolano.

Nella letteratura antropologica esistono alcuni studi che riguardano le piante che marcano i confini: Paul Sheridan chiama queste fasce vegetative polymarcation plants, perché rivestono più funzioni e si collocano proprio all'intersezione di dinamiche sociali, materiali e simboliche (Sheridan 2016). Forse l'esempio più noto di quanto intende Sheridan quando parla di polymarcation plants è rappresentato dallo studio condotto da Roy Rappaport sulle dinamiche di equilibrio ecologico tra gli tsembaga maring della Papua Nuova Guinea in Maiali per gli antenati (1980). Considerando il villaggio come il punto di partenza della sua analisi, Rappaport analizza i flussi di energia calorica (in modo specifico il taro e il maiale) all'interno del sistema. Quando la 
popolazione di maiali diventa troppo numerosa e scappa, invadendo gli orti di un altro villaggio, i conflitti, che normalmente rimangono ad un livello gestibile, si fanno più drammatici e arrivano a scontri a colpi di ascia. Gli uomini, per rimarcare che oramai sono in guerra, sradicano la cordilina che era stata piantata nei luoghi di pace un decennio prima, generalmente per sancire l'inizio del kaiko, un rito di uccisione sacrificale di un certo numero di maiali in onore degli antenati. Dopo aver lasciato le loro case, a loro volta marcate dalla presenza della cordilina, i guerrieri e i loro alleati vanno in battaglia.

13 Alla fine del conflitto, ambo le parti riposizionano le piante sui loro confini e le usano per marcarne di nuovi, dopodiché la pace è ristabilita. Rappaport interpreta l'atto di sradicare e ripiantare la cordilina come una sorta di "trasduttore" rituale che permette al sistema socio-ecologico di far fronte e di adattarsi ai cambiamenti nella popolazione di maiali e di persone, di redistribuire queste ultime sul territorio, di determinare la frequenza e intensità della violenza e creare relazioni di scambio tra gli tsembaga e i loro alleati (Rappaport 1980). Se il paesaggio tsembaga fosse un testo scritto, la cordilina sarebbe la sua punteggiatura perché è radicata, letteralmente, alla confluenza dell'economia agricola, della politica acefala, della pratica rituale, della religione e delle relazioni di genere. Rappaport si concentra in particolare sul kaiko come meccanismo per contrastare la competitività della produzione suina, quando l'aumento della stessa incide negativamente sul rapporto tra proprietari degli orti confinanti e i maiali, ma la cordilina è chiaramente un oggetto di confine multivocale che demarca diversi aspetti e ambiti (Ibidem).

14 All'epoca in cui Rappaport pubblica Maiali per gli antenati, così come emerge da altri studi condotti negli ultimi due decenni del secolo scorso ${ }^{1}$, le piante sono ormai inserite in un discorso più ampio, definito "la svolta spaziale": si tratta di un approccio maturato sulla scia di eventi socio-politici di portata globale come la fine della Guerra Fredda e la destabilizzazione geopolitica mondiale - che ha avuto per oggetto un ripensamento dei concetti di spazio, luogo e paesaggio. Nella riflessione degli autori che hanno lavorato sulla produzione dello spazio e sulla transizione da spazio a luogo, le piante da confine si delineano come entità relazionali che «fanno cose» (Sheridan 2016: 36): si posizionano al centro di spazi contestati (Low 1999), di luoghi di memoria (Nora 1984-1992), di processi di de-territorializzazione e ri-territorializzazione (Gupta, Ferguson 1997), in una parola, sono una parte decisiva nella formazione del «senso dei luoghi» (Feld, Basso 1996: 14).

Sulle piante che marcano i confini, sulle loro relazioni con altre specie - in questo caso api e umani - e, più in generale, sulle specie vegetali che "ripopolano" i paesaggi, è articolato il mio contributo. Esso riprende un lavoro, iniziato nel 2017 nell'ambito di un progetto di ateneo finanziato dalla Fondazione San Paolo di Torino (2015-2017) ${ }^{2}$ sulla figura dei nuovi coltivatori in area alpina, in particolar modo tra la media e l'alta Valle Susa, in provincia di Torino ${ }^{3}$. Con l'avanzare del progetto avevo iniziato ad interessarmi ad altri attori sociali coinvolti nel recupero delle colture alpine, scoprendo che alcuni di loro, oltre a possedere i terreni dove erano seminate le specie interessate, erano apicoltori $o$, in altri casi, sui loro appezzamenti erano posizionate le arnie. Se inizialmente mi ero proposta di comprendere cosa distingue un apicoltore stanziale da uno nomade e se la categoria di "domestico" e "selvatico" potesse applicarsi alle api mellifere (cfr. Zola 2017), per questo contributo ho ripreso contatti con alcuni di loro, effettuando delle interviste di consolidamento e aggiornamento. In particolar modo ho 
rivisto alcuni soci dell'Associazione Apicoltori Alta Valle Susa, che praticano apicoltura stanziale compresa tra un'altitudine di 1000-1400 msldm nei dintorni del Comune di Oulx (TO). Ho nuovamente raggiunto, inoltre, tre apicoltori: uno di loro, G.T., risiede a Ceresole d'Alba (TO), nella pianura in prossimità di Torino e nei mesi estivi trasferisce le sue arnie in quota, prevalentemente sui pascoli della Valle Argentera o presso l'Alpe Laune, poco sopra il Comune di Sauze d'Oulx, in zone vicine all'attività apistica portata avanti dall'Associazione Apicoltori Alta Valle Susa. Gli altri due, A.G. e L. D.R., marito e moglie, abitano a Camerano, una zona collinare della provincia di Asti e praticano apicoltura nomade a $1455 \mathrm{~m}$. a Prali, in Val Germanasca ${ }^{4}$.

Durante le visite alle zone in cui gli apicoltori citati operano, dalle interviste condotte o da semplici conversazioni sia nel 2017 sia nelle mie ultime discese sul campo, un tema è emerso con particolare rilievo: l'impoverimento del paesaggio. Questo, dalle loro parole, si configura come uno degli esiti più manifesti di una serie di azioni reiterate nel tempo, quali l'uso indiscriminato delle risorse, la sempre minore conoscenza del territorio e dei rischi ad esso connessi, l'utilizzo di pesticidi, particolarmente diretti alle monocolture. Ciò ha avuto come effetto diretto la diminuzione delle api e una sempre minore varietà di piante sulle quali esse bottinano.

Tutto ciò apparve già molto evidente quando, nel 2017, visitai per la prima volta la cascina di G.T. a Ceresole d'Alba. Dalla sua abitazione-laboratorio si apriva un panorama piuttosto comune nelle aree di pianura pedemontane piemontesi: intere distese di coltivazioni di mais, intervallate ogni tanto da appezzamenti prativi che sembravano incolti e da file di vegetazione che punteggiavano il paesaggio. Ciò che $\mathrm{mi}$ aveva colpita già allora, era il fatto che i prati non erano affatto incolti o abbandonati e gli alberi e le siepi che delimitavano i confini tra i campi di mais non erano lì da sempre: erano entrambi opera di G.T. che, nel tempo, aveva effettuato una sorta di "rinselvatichimento" delle aree in cui le sue api bottinavano, per contrastare almeno in parte alcuni dei problemi sopraccitati. Ma quali erano e sono le piante sulle quali le api bottinano? Perché si rende necessaria un'opera di "rinselvatichimento" e di "ripopolamento" del paesaggio?

\section{Quando umani, api e piante si incontrano}

L'ape "domestica", Apis mellifera mellifera, è ampiamente presente sul territorio italiano dove, in tempi immemorabili, si diffuse dall'Asia giungendo fino all'attuale Pianura Padana, trovando un ambiente adatto alla sua sopravvivenza durante il periodo delle glaciazioni. La sua presenza in Italia, avvenuta in epoche così lontane, sta ad indicare che già allora esistevano fonti di nutrimento rappresentate da piante nettarifere $\mathrm{e}$ pollinifere che assicuravano cibo sufficiente al sostentamento delle famiglie di api (Ferrazzi 1982).

19 Norme riguardanti l'allevamento o la proprietà degli sciami, il commercio del miele, le multe per i furti di alveari o prodotti apistici sono contenute in documenti dei secoli XIII, XIV e XV rinvenuti nel cuneese (a Villafalletto, Busca, Murazzano), nel torinese (a Lessolo, Strambino), nell'alessandrino (a Gavi, Pareto e Casale) e nel territorio ossolano, a Vogogna. Queste notizie, anche se molto scarse, documentano che l'apicoltura in Piemonte era diffusa sin dalla fine del Medio Evo (Marletto 1982). È altresì attestata, negli Statuti Comunali piemontesi dei secoli XIV e XV, l'esistenza di siepi vive con funzioni anche mellifere chiamate clausurae, che chiudevano appezzamenti di terra. 
Erano poste al confine di coltivazioni intensive come vigne, alteni, airali, ma anche orti e piccoli appezzamenti coltivati a cereali (Panero 1994).

Fino alla Seconda Guerra Mondiale l'attività apistica, pur rimanendo sempre complementare a quella agricola, costituiva parte integrante della vita quotidiana dei contadini; era una pratica estensiva e diffusa su tutto il territorio regionale. Con l'avvento dell'industrializzazione, della progressiva meccanizzazione delle tecniche agricole e l'esodo dalle campagne, si assistette ad un calo dell'attività. All'inizio degli anni ' 80 del secolo scorso, tuttavia, alcuni autori testimoniavano che già da qualche decennio si era registrata una ripresa che assumeva però forme diverse rispetto al passato: impostata su basi scientifiche di alta specializzazione, privilegiava in molti casi l'allevamento intensivo, reso possibile sia dall'aumentata remuneratività del prodotto, sia dalla maggiore richiesta dovuta anche ad un ritorno, talora enfatizzato, verso $\mathrm{i}$ prodotti naturali (Giuliano, Novaro Pulcher 1982). Negli ultimi decenni l'attenzione verso le api e l'apicoltura è in crescita, dettata spesso dalla preoccupazione per la sorte delle api, soggette negli anni scorsi a morie molto intense (Coyaud 2008).

21 Uno dei principali fattori segnalati sia dai progetti europei dedicati al monitoraggio degli insetti impollinatori ${ }^{5}$ sia dagli interlocutori che ho ascoltato, è rappresentato dall'indebolimento dell'habitat per il foraggiamento delle api, a sua volta provocato dal cambiamento nell'uso della terra e del paesaggio a partire dalla Seconda Guerra Mondiale: gli ecosistemi ideali per le api sono diminuiti e si sono ridotte anche le zone semi-naturali.

Questo dato trova conferma nelle parole di A.G. e L.D.R, che fanno direttamente riferimento a come sia cambiata la gestione del territorio e, di conseguenza, l'equilibrio tra zone coltivate e boschive:

Qui i boschi non vengono più tagliati, queste piante [le acacie, n.d.a.] sono tutte vecchie e non ci sono più nemmeno le piante di castagno e tiglio che hanno tolto quando hanno messo le vigne, poi le vigne si sono perse e sono state abbandonate e sono tornati i boschi ma non con piante mellifere. L'unica è l'acacia che produce molto nettare ma se non viene tagliata non fiorisce abbastanza per produrre nettare e poi muore... prima erano i contadini che facevano il bosco per la legna da ardere ecc... adesso nei nostri boschi la pianta più forte si impone e adesso ad esempio viene prima l'edera, i rovi eccetera, tutte piante infestanti che uccidono il bosco (A.G. e L.D.R, Camerano/Prali, intervista di giugno 2021).

Analogamente, dagli studi di paleobotanica è emerso che nell'Italia del Nord, a partire dal periodo postglaciale fino a tempi relativamente recenti, esistevano grandi estensioni di foreste sia nella Pianura Padana, dove prevalevano le querce, gli olmi e tigli, sia sulle montagne circostanti, ricoperte di piante in prevalenza resinose. La flora dell'Italia del Nord, per lungo tempo rimasta quasi invariata, negli ultimi secoli ha subito forti cambiamenti dovuti all'opera dell'uomo: nel 1982 Paola Ferrazzi riportava infatti che l'aumento della coltura del granturco o mais, coltivata in vaste superfici, aveva sottratto notevole spazio a zone prative, boschive o incolte perlopiù ricche di piante di interesse apicolo. Analogamente, l'incremento della cerealicoltura e in particolare del riso aveva «eliminato l'apicoltura da ampie zone sia per la distruzione quasi completa degli ambienti naturali, sia per i danni arrecati con le fortissime dosi di diserbante usate sulla flora residua» (Ferrazzi 1982: 211).

Un altro fattore che in larga parte contribuisce alla moria delle api e che è in stretta correlazione con la questione del foraggiamento, come affermava Ferrazzi, è relativo ai trattamenti chimici, segnalati come responsabili delle morie dall'Istituto di Apicoltura 
dell'Università di Torino già più di trent'anni fa. Un gruppo di biologi, neurobiologi, entomologi dell'Università di Torino, in un articolo del 2013 ha constatato che dosi subletali di neonicotinoidi, insetticidi molto utilizzati in agricoltura, sono in grado di interferire negativamente sulla capacità di orientamento delle api (Laurino et al. 2013). Questo dato trova conferma nelle parole di G.T. di Ceresole d'Alba:

Il girasole ad esempio è un'ottima pianta da miele, ma se fanno i trattamenti diventa pericolosa. Vedi ad esempio i girasoli grossi, da olio, le api vanno a succhiare il nettare ma le stordisce... quando rientrano nell'alveare sono stordite, il veleno agisce sul sistema nervoso (G.T., Ceresole d'Alba/ Alpe Laune, interviste di maggio 2017 e giugno 2021).

I trattamenti chimici sono anche utilizzati nei piccoli appezzamenti coltivati come gli orti, provocando, come testimoniato da A.G. e L.D.R., la moria dei nuclei famigliari:

Qui a Camerano c'è una valle dove molte persone hanno gli orti, la chiamiamo la valle degli orti. Anche mio papà ha l'orto là. È successo che abbiamo messo un nucleo là e questo nucleo non è mai durato. Sicuramente chi fa l'orto mette dei prodotti chi per le cimici, chi per le lumache, sono tutti prodotti chimici, le persone anziane fanno così, due tre volte abbiamo provato e le api sono sempre morte (A.G. e L.D.R., Camerano/Prali, intervista di giugno 2021).

In alta quota, sebbene la minaccia dei pesticidi sia meno sentita rispetto alla pianura, l'impoverimento del suolo, causato da sbalzi climatici troppo repentini, secondo gli apicoltori influisce negativamente sulla salute delle api:

Quest'anno a marzo faceva caldo e hanno iniziato a muoversi, poi freddo a maggio, qui le prime fioriture sono gelate tutte, i ciliegi selvatici, i pruni sono gelati... Certi fiori che normalmente fioriscono ad agosto, a fine giugno erano già fioriti. Ma anche a marzo-aprile faceva troppo caldo, la campagna non è pronta e poi i fiori vengono brutti, corti, non hanno nettare, se fioriscono ad aprile invece che a giugno non va bene, e alla fine il freddo deve comunque arrivare (A.M. e L.F., Sauze di Cesana, Associazione Apicoltori Alta Valle Susa, intervista di giugno 2021).

Anche la situazione attuale dei pascoli è un problema serio per la raccolta di polline:

Un'altra cosa che per noi è un danno enorme è il pascolo anticipato sul territorio. Abbiamo il pascolo dei caprioli e dei cervi che una volta non c'erano, dopo la neve cominciano a rosicchiare tutto. Un buon raccolto del miele di montagna qui era la lupinella, che se mangiata presto non cresce più. Tra 10-15 anni avremo solo più erba che non dà fiori...ci sono anche i margari ${ }^{6}$ che vengono su sempre più presto (L.F., Bousson, Associazione Apicoltori Alta Valle Susa, intervista di giugno 2021).

Questo si ricollega ad una delle prime cause menzionate precedentemente, ossia la mancanza di habitat per il foraggiamento. Sempre secondo le parole di un apicoltore dell'Associazione Apicoltori Alta Valle Susa: «Una volta i terreni erano anche coltivati a segale, erano concimati, adesso le mucche passano sempre nella stessa zona, nessuno toglie le pietre, non ci sono più fiori anche per questo motivo qua» (A.M., Sauze di Cesana, Associazione Apicoltori Alta Valle Susa, intervista di maggio 2017).

Il quadro tracciato evidenzia che la gestione del territorio è frutto di un equilibrio complesso tra diversi attori, tra cui rientrano anche quelli umani. In tal senso, sono esemplificative le parole di L.D.R:

C'era una cultura dietro... c'erano le vigne e c'era bisogno di pali per le vigne che dovevano essere di $12 \mathrm{~cm}$ di diametro quindi le piante, quando raggiungevano quella dimensione lì, venivano tagliate per cui c'era un rinnovo di piante e le piante non venivano vecchie. Non c'erano boschi ma si faceva il miele, adesso ci sono i boschi ma non si fa più il miele (L.D.R., Camerano/Prali, intervista di giugno 2021). 


\section{Ripopolare, rinselvatichire}

minore, l'eccessivo uso di pesticidi, è dato dalla possibilità di seminare nei terreni agricoli specie spontanee, in modo da fornire nutrimento variegato per le api, ma anche per altri insetti che rivestono il ruolo di impollinatori selvatici. Le piante e le erbe spontanee che vengono reintrodotte, spesso nella forma di siepi lineari, vengono definite fasce vegetali o fasce tampone (quest'ultimo termine è maggiormente riferito a parti di vegetazione frapposte tra i coltivi e i corsi d'acqua) dall' Istituto Superiore per la Protezione e la Ricerca Ambientale (ISPRA). Sono di grande utilità e, secondo le ultime due guide selvicolturali divulgate dalla Regione Piemonte, «sono in grado di esercitare diverse funzioni ambientali» (Regione Piemonte 2018a: 5) ${ }^{7}$. In particolare quelle ripariali, che si trovano lungo i corsi d'acqua o vicino ai laghi, proteggono le sponde dagli smottamenti, migliorano le condizioni dei corsi d'acqua, aumentano la biodiversità dell'ecosistema, attirando e favorendo la diffusione di diverse specie animali contribuendo alla «connessione dell'ecosistema» (Ibidem: 7).

Si tratta dunque di una vera e propria attività di "rinselvatichimento" dell'ambiente circostante ma controllato, gestito dagli esseri umani sia a livello istituzionale, sia tramite azioni concrete sul territorio. Se oggi attraverso le guide tecniche che fanno capo ai Piani di Sviluppo Rurale è possibile conoscere nel dettaglio quali specie introdurre, dove, a quale distanza le une dalle altre, per quali finalità (ad esempio produzione legnosa, mellifera, edule), molto prima che i Piani di Sviluppo Rurale entrassero in vigore, G.T. aveva già iniziato la sua opera di "rinselvatichimento" attraverso l'impianto di diverse specie, tra cui principalmente la rosa canina (Rosa Canina) e il biancospino (Crataegus Levigata):

Io ho 21 ettari di terra e in circa 10 sto cercando di mettere delle piante, ad esempio negli spazi vuoti ho cercato di mettere del biancospino [Crataegus Levigata, n.d.a.]... sto cercando nel piccolo perché le api hanno un raggio di azione di $3 \mathrm{~km}$, di qui vanno a Ceresole, quindi 10 ettari non sono molti, però è già qualcosa (G.T., Ceresole d'Alba/Alpe Laune, intervista di maggio 2017 e di giugno 2021).

G.T. ha agito su due fronti: da un lato ha ricreato i prati stabili (a cui facevano riferimento poc'anzi gli apicoltori dell'alta Valle Susa lamentandone la graduale scomparsa), seminando specie prative che generalmente crescono spontanee e che sono particolarmente apprezzate:

... Una volta c'erano i prati stabili che erano una bella cosa per le api perché c'erano sempre dei fiori, adesso sto cercando di ricreare i prati stabili con tarassaco [Taraxacum Officinale, n.d.a.], ligustro [Ligustrum Vulgare, n.d.a.] e corniolo [Cornus Mas, n.d.a.], trifoglio [Trifolium, n.d.a.] e facelia [Phacelia Tanacetifolia, n.d.a.] (G.T., Ceresole d'Alba/Alpe Laune, intervista di giugno 2021).

Dall'altro lato ha invece impiantato delle siepi di specie arbustive spontanee, sistemandole sui confini dei suoi appezzamenti e sfruttando il fatto che alcune di esse si trovavano già sui suoi terreni:

Ai confini dei miei appezzamenti c'erano già delle piante di prugnolo [Prunus Spinosa, n.d.a.] e di biancospino [Crataegus Levigata, n.d.a.] ma nel tempo ho piantato altre specie tra cui appunto la rosa canina, l'acero campestre [Acer Campestre, n.d.a.], il tiglio, fino a specie non autoctone come la piracanta [Pyracantha Coccinea, n.d.a.], oltre alle acacie. Molte di queste piante (che sono praticamente tutte spontanee) le ho comprate e ne ho fatto delle siepi da mettere lungo i confini dei miei campi (G.T., Ceresole d'Alba/Alpe Laune, intervista di giugno 2021).

Archivio antropologico mediterraneo, Anno XXIV, n. 23 (2) | 2021 
dunque l'importanza delle piante che segnano i confini come entità di raccordo di specie diverse (foto 1 ), soprattutto in un contesto che è profondamente mutato negli ultimi decenni: secondo i due report della Regione Piemonte, infatti, nell'arco di circa 30 anni le siepi campestri sono passate da uno sviluppo medio di $100 \mathrm{~m} / \mathrm{ha}$ (100 ettari) a 10m/ha, con una perdita del 90\% (Regione Piemonte 2018b).

Se un tempo le siepi e le alberature che delimitavano i confini tra i campi coltivati erano molto diffuse, con l'avvento dell'agricoltura intensiva, sempre più caratterizzata dalla meccanizzazione ma anche da superfici che sono state accorpate in appezzamenti di dimensioni sempre più ampie, le siepi sono gradualmente ma altrettanto drasticamente scomparse: «Oggi c'è la tendenza a togliere le siepi perché danno fastidio ai trattori che devono passare, sono scomode per i contadini, ma in realtà servono» (G.T., Ceresole d'Alba/Alpe Laune, intervista di giugno 2021). reti di gestione meccanizzata dei corsi d'acqua o l'allargamento delle strade di campagna. Questo ha comportato anche la chiusura, attraverso tubazioni interrate, dei fossi, elementi estremamente utili perché, uniti a fasce arbustive, evitavano smottamenti e allagamenti. Queste condizioni, protratte nel tempo, hanno avuto effetti dannosi su tutto l'ecosistema, a partire proprio dalla diminuzione di alcune specie di piante arbustive come i gelsi, i cornioli, gli olmi, i tigli, per continuare con i mammiferi, gli anfibi, gli uccelli che vi trovavano rifugio, senza ovviamente dimenticare gli insetti impollinatori, che utilizzavano queste fasce vegetali come fonte di nutrimento.

37 A differenza di G.T., A.G. e L.D.R. a Camerano tengono le loro arnie in zone collinari: sebbene sul territorio dove le api bottinano siano già presenti diversi arbusti e alberi spontanei, questi non sono melliferi. Di conseguenza i due apicoltori hanno avviato anche loro un'opera di "ripopolamento", nell'ottica di avere specie con tempi di fioritura diversi:

Noi abbiamo un pezzo di collina verso la cima e uno in basso e adesso abbiamo comprato anche quello in mezzo dove metteremo intanto delle mandorle che fioriscono presto, così diamo da mangiare anche a loro [le api, n.d.a.]: con una decina di piante di mandorle non fai il miele ma almeno dai loro da mangiare... poi erba medica [Medicago Sativa, n.d.a.]... volevamo anche mettere i topinambur [Helianthus Tuberosus, n.d.a.] che fioriscono per ultimi e poi abbiamo un pezzo dove vorremmo mettere dei castagni. Ci sono tanti progetti dove mettono i castagni per non fare solo più apicoltura di nomadismo ma stabile, anche se non è facile (A.G. e L.D.R, Camerano/Prali, intervista di giugno 2021).

Uno degli aspetti più interessanti di questa opera di "rinselvatichimento" e "ripopolamento controllato", a mio parere, è che nei documenti come le guide tecniche della Regione Piemonte è utilizzato un lessico che a tutti gli effetti richiama l'importanza dell'approccio multispecie: i report regionali parlano ad esempio di queste fasce vegetali come di ecotoni, ovvero «habitat di transizione tra ecosistemi adiacenti di natura diversa» (Regione Piemonte 2018b: 7) o, ancora, riferendosi agli insetti impollinatori, affermano che «tra gli abitanti "utili" delle formazioni lineari si ricordano ancora gli insetti pronubi (Imenotteri, Ditteri e Lepidotteri), che con l'impollinazione consentono la riproduzione di specie vegetali, entomofile, naturali o coltivate» (Ibidem: 8). Analogamente, l'ultimo rapporto dell'ISPRA sugli impollinatori, utilizza concetti quali «simbiosi mutualistica, interazione mutualistica» (2021:16) tra insetti pronubi e specie vegetali.

Archivio antropologico mediterraneo, Anno XXIV, n. 23 (2) | 2021 
In ultima analisi, queste fasce vegetali che marcano i confini sono definite «corridoi di connessione, foreste lineari» (Ibidem: 8), intendendo che sono utili a specie diverse per scopi differenti: a quella umana che può beneficiare della legna, dei frutti spontanei e del miele, a quella non-umana animale che può trovare in esse rifugio ma anche nutrimento, a quella non-umana vegetale stessa, che è riparata da vento ed esondazioni. Bellucci, Piotto e Sili (2021) definiscono i corridoi di connessione come un insieme di nodi e linee. I nodi sono rappresentati dagli spazi graditi oppure occupati stabilmente da uccelli e insetti impollinatori, mentre le linee, come le foreste lineari o le alberature che delimitano i confini, mettono in connessione i nodi tra loro. Le piante che marcano i confini, in tal senso, agiscono da raccordo, da anello di congiunzione nelle relazioni tra specie diverse.

\section{Conclusioni}

Se le riflessioni mosse dalla "svolta ontologica" e dalla prospettiva multispecie sono state applicate in modo particolare alle popolazioni amerindiane e, in generale, a quelle extraeuropee, esistono ad oggi ancora poche testimonianze su come esse siano state recepite dagli attori sociali in un contesto occidentale ed europeo, soprattutto alla luce dei grandi cambiamenti di natura politica, ma soprattutto ambientale, che hanno caratterizzato gli ultimi due decenni.

41 Il caso di studio da me presentato non solo si presta particolarmente ad essere analizzato secondo una prospettiva multispecie, ma dimostra che gli esseri umani che "compartecipano" al processo di rinselvatichimento e ripopolamento del paesaggio sono sì attanti, come direbbe Latour, ma lo sono anche e soprattutto le piante e in particolare quelle che marcano i confini. Le loro caratteristiche proprie e peculiari, come avere le spine o essere tossiche o velenose, le rendono completamente avviluppate, per usare una terminologia prettamente vegetale, in reti di interdipendenza ecologica (Hodder 2012). Questo è ben espresso nelle narrazioni dei miei interlocutori: da un lato hanno evidenziato le motivazioni e le pratiche che hanno portato ad un impoverimento del paesaggio, dall'altro i tentativi da parte loro a impegnarsi in un'opera di "rinselvatichimento" e di "ripopolamento" delle aree dove bottinano le loro api, evidenziando ulteriormente un approccio dove gli umani, come afferma Jacques Tassin, si collocano in una linea di continuità con le altre specie viventi (2018).

Ancora una volta si potrebbe affermare che le piante non sono solo "buone da pensare" ma "sono buone da pensare con" (Haraway 2008), perché la loro agency riflette e modella i rapporti sociali che si creano in sistemi ecologici differenti (Sheridan 2016).

In ultima analisi è necessario prendere in considerazione un'altra forma di interdipendenza: quella tra lessici specialistici, nel mio caso selvicolturali e antropologici che si configura anch'essa come una continuità tra pratiche e saperi, di grande rilevanza per i possibili sviluppi della ricerca in questo senso (Cuturi 2020). Come accennato in precedenza, concetti come "connessione dell'ecosistema", "corridoi di connessione" anche nelle scienze sociali sono entrati a pieno titolo nel dibattito sulla svolta ontologica, richiamando in più parti sia il concetto di abitare "lungo" di cui parla Ingold ma anche quello di rete di relazioni, dove gli attori sociali sono parte di nodi più complessi e interdipendenti tra loro. Collaborazione, relazionalità e interdipendenza tutte caratteristiche che sono proprie dell'essere pianta - possono cambiare il modo in 
cui pensiamo alle dinamiche sociali (Lewis-Jones 2016). Quando si ragiona sulle piante diventa quindi di primaria importanza "espandere i termini della relazione» (Gallagher, DiNovelli-Lang 2014: 3), soprattutto in un momento storico come quello che stiamo vivendo.

\section{BIBLIOGRAFIA}

Archambault J.S., 2016 «Taking Love Seriously in Human-Plant Relations in Mozambique», in Cultural Antropology, 31, II: 244-271.

Balick M. (ed.), 2009 Ethnobotany of Pohnpei, University of Hawaii Press, Honolulu.

Bellucci V., Piotto B., Sili V. (a cura di), 2021 Piante e insetti impollinatori: un'alleanza per la biodiversità, ISPRA, serie e rapporti, 350/2021.

Berrigan C., 2014 «Life Cycle of a Common Weed», in E. Kirksey (ed.), The Multispecies Salon, Duke University Press, Durham, NC: 165-180.

Bonato L. (a cura di), 2017 Aree Marginali. Sostenibilità e saper fare nelle Alpi, FrancoAngeli, Milano. Cassidy R., Mullin M. (eds.), 2007 Where the Wild Things Are Now: Domestication Reconsidered, Berg, Oxford.

Coyaud S., 2008 La scomparsa delle api, Mondadori, Milano.

Cuturi F., 2020 «Al di là della svolta botanica. Continuità tra pratiche di vita e di pensiero scientifici e nativi», in F. Cuturi (a cura di), La natura come soggetto di diritti: prospettive antropologiche e giuridiche a confronto, EditPress, Firenze: 219-286.

Descola P., 1992 «Societies of nature and the nature of society», in A. Kuper (ed.), Conceptualizing Society, Routledge, London and New York: 107-127.

Descola P., 1996 In the society of nature: a native ecology in Amazonia, Cambridge University Press, Cambridge.

Descola P., 2000 «L'Anthropologie de la Nature », in Annales. Histoire, Sciences Sociales, 1 (Jan-Fev): 9-25.

Descola P., 2020 Oltre natura e cultura, Raffaello Cortina, Milano, trad. di Par delà de nature et culture, Gallimard, Paris, 2005.

Feld S., Basso K., 1996 Senses of Place, School of American Research Press, Santa Fe.

Ferrazzi P., 1982 «Evoluzione della flora apistica», in C. Vidano, A. Arzone, F. Marletto, S. Bizzarri (a cura di), Per un museo dell'Agricoltura in Piemonte III. Passato e presente dell'apicoltura Subalpina, Turingraf, Torino: 205-218.

Gallagher P., DiNovelli-Lang D., 2014 «Introduction: Nature and Knowledge-Contemporary Ecologies of Value», in Environment and Society: Advances in Research, 5, I: 1-6.

Gershon I., 2010 «Bruno Latour (1947-)», in J. Simons (ed.), From Agamben to Žižek, Edinburgh University Press, Edinburgh: 161-176. 
Giuliano W., Novaro Pulcher A., 1982 «Reperti apistici nei musei locali piemontesi », in C. Vidano, A. Arzone, F. Marletto, S. Bizzarri (a cura di), Per un museo dell'Agricoltura in Piemonte III. Passato e presente dell'apicoltura Subalpina, Turingraf, Torino: 41-62.

Gupta A., Ferguson J. (eds.), 1997 Culture, Power, Place, Duke University Press, Durham.

Haraway D., 2008 When species meet, University of Minnesota Press, Minneapolis.

Haraway D., 2016 Staying with the trouble: Making kin in the Chtulucene, Duke University Press, Durham.

Hartigan J., 2015 «Plant Publics: Multispecies Relating in Spanish Botanical Gardens», in Anthropological Quarterly, 88, II: 481-507.

Henare A. et al., 2006 «Introduction: thinking through things», in A. Henare, M. Holbraad, S.Wastell (eds.), Thinking through things: theorising artefacts ethnographically, Routledge, London: $1-31$.

Holbraad M., 2009 «Ontography and alterity: defining anthropological truth», in Social Analysis, 53: 80-93.

Hodder I., 2012 Entangled, Wiley-Blackwell, West Sussex.

Hustak C., Myers N., 2012 «Involutionary Momentum: Affective Ecologies and the Sciences of Plant/Insect Encounters», in Differences, a Journal of Feminist Cultural Studies, 25, V: 74-118.

Ingold T., 2000 The Perception of the Environment. Essays on Livelihood, Dwelling and Skill, Routledge, London.

Ingold T., 2015 The life of Lines, Routledge, London.

Kirksey S.E., Helmreich S., 2010 «The Emergence of Multispecies Ethnography», in Cultural Anthropology, 25, IV: 545-576.

Kohn E., 2007 «How Dogs Dream: Amazonian Natures and the Politics of Transspecies

Engagement », in American Ethnologist, 34, I: 3-24.

Kohn E., 2013 How Forests Think, University of California Press, Berkeley.

Kramer A., Havens K., 2015 «Report in Brief: Assessing Botanical Capacity to Address Grand Challenges in the United States», in Natural Areas Journal, 35, I: 83-89.

Latour B., 2004 Politcs of Nature, Harvard University Press, Cambridge, trad. di Politiques de la Nature, La Decouverte, Paris, 1999.

Latour B., 2005 Reassembling the Social: An Introduction to Actor-Network Theory, Oxford University Press, Oxford.

Latour B., 2014 «Anthropology at the Time of the Anthropocene», in New Literary History, 45:1-18.

Laurino D. et al., 2013 «Toxicity of neonicotinoid insecticides on different honey bee genotypes», in Bulletin of Insectology, 66, I: 119-126.

Lewis-Jones K., 2016 «Introduction. People and Plants», in Environment and Society: Advances in

Research, 7: 1-7.

Low S., 1999 Theorizing the City, Rutgers University Press, New Brunswick, NJ.

Mancuso S., 2017 Plant Revolution, Milano-Firenze, Giunti.

Marder M., 2013 Plant Thinking: A Philosophy of Vegetal Life, Columbia University Press, New York. 
Marletto F., 1982 «Inizio ed evoluzione dell'apicoltura razionale in Piemonte», in C. Vidano, A. Arzone, F. Marletto, S. Bizzarri (a cura di), Per un museo dell'Agricoltura in Piemonte III. Passato e presente dell'apicoltura Subalpina, Turingraf, Torino: 220-226.

Moore S.F., 1986 Social Facts and Fabrication, Cambridge University Press, Cambridge.

Nora P. (éd.), 1984-1992 Les lieux de mémoire, Gallimard, Paris.

Ogden L.A. et al., 2013 «Plants, People and Things», in Environment and Society: Advances in Research, 4: 5-24.

Panero F., 1994 Strutture del mondo contadino, Gribaudo, Cavallermaggiore.

Perullo N., 2016 «Piedi, linee, tempo, labirinto. L'ecologia della vita come corrispondenza a partire da Tim Ingold», in DIANOIA, 23:399-417.

Porporato M., 2014 «L'avanzata della vespa velutina, pericolo per le api», in Ecoscienza, 4:26-27.

Rappaport R., 1980 Pigs for the Ancestors, Yale University Press, New Haven ,CT.

Regione Piemonte, 2018 a Le fasce tampone vegetate riparie. Realizzazione e gestione, Centro Stampa della Regione Piemonte, Torino.

Regione Piemonte, $2018 \mathrm{~b}$ Le fasce tampone vegetate riparie arbustive-arboree. Realizzazione e gestione, Centro Stampa della Regione Piemonte, Torino.

Rival L., 2007 «Domesticating the Landscape, Producing Crops and Reproducing Society in Amazonia», in D. Parkin, S. Uljijaszek (eds.), Holistic Anthropology: Convergence and Emergence, Berghahn Books, New York and Oxford: 72-90.

Rival L., 2016 «Botanical Ontologies Special Section of the Journal of Ethnobiology Post-Face», in Journal of Ethnobiology, 36, II: 147-149.

Sheridan M., 2016 «Boundary Plants, the Social Production of Space, and Vegetative Agency in Agrarian Societies», in Environment and Society: Advances in Research, 7: 29-49.

Smith P. et al., 2011 «Making the Case for Plant Diversity», in Seed Science Research, 21, I: 1-4.

Stoetzer B., 2018 «Ruderal Ecologies: Rethinking Nature, Migration, and the Urban Landscape in Berlin, in Cultural Anthropology, 3, II: 295-323.

Stépanoff C., 2021 L'animal et la mort, Paris, La Découverte.

Stone G., 1994 «Agricultural Intensification and Perimetrics: Ethnoarchaeological Evidence from Nigeria», in Current Anthropology, 35, III: 317-324.

Strathern M., 1995 «The nice thing about culture is that everyone has it», in M. Strathern (ed.), Shifting contexts: transformations in anthropological knowledge, Routledge, London: 153-173.

Tassin J., 2018 Come pensano le piante, Milano, Sonda edizioni.

Tsing A., 1995 «Empowering Nature, or: some Gleanings in Bee Culture», in S. Yanagisako, C. Delaney (eds.), Naturalising Powers: Essays in Feminist Cultural Analisys, Routledge, New York: 113-143.

Tsing A., 2015 The Mushroom at the End of the World: on the Possibility of Life in Capitalist Ruins, Princeton University Press, Princeton.

Viveiros de Castro E., 1998 «Cosmological Dexis and Amerindian Perspectivism», in Journal of the Royal Anthropological Institute, 4, III: 469-488. 
Viveiros de Castro E. et al., 2014 The Politics of Ontology. Theorizing the Contemporary, «Cultural Anthropology», online version: https://culanth.org/fieldsights/series/the-politics-of-ontology

Viveiros de Castro E., 2015 «Who is Afraid of the Ontological Wolf?: Some Comments on an Ongoing Anthropological Debate», in The Cambridge Journal of Anthropology, 33 I: 2-17.

Zeder M., 2006 «Central Questions in the Domestication of Plants and Animals», in Evolutionary Anthropology, 15: 105-117.

Zola L. (a cura di), 2017 Ambientare. Idee, saperi, pratiche, FrancoAngeli, Milano.

Zola L., 2017 «Storie di apicoltura, cambiamenti climatici e resilienze», in L. Zola (a cura di), Ambientare. Idee, saperi, pratiche, FrancoAngeli, Milano: 234-246.

Wandersee J., Schussler E.E., 2001 «Toward a Theory of Plant Blindness», in Plant Science Bulletin, 47, I: $2-8$.

West P. 2006, Conservation is Our Government, Duke University Press, Durham, NC.

\section{ALLEGATO}

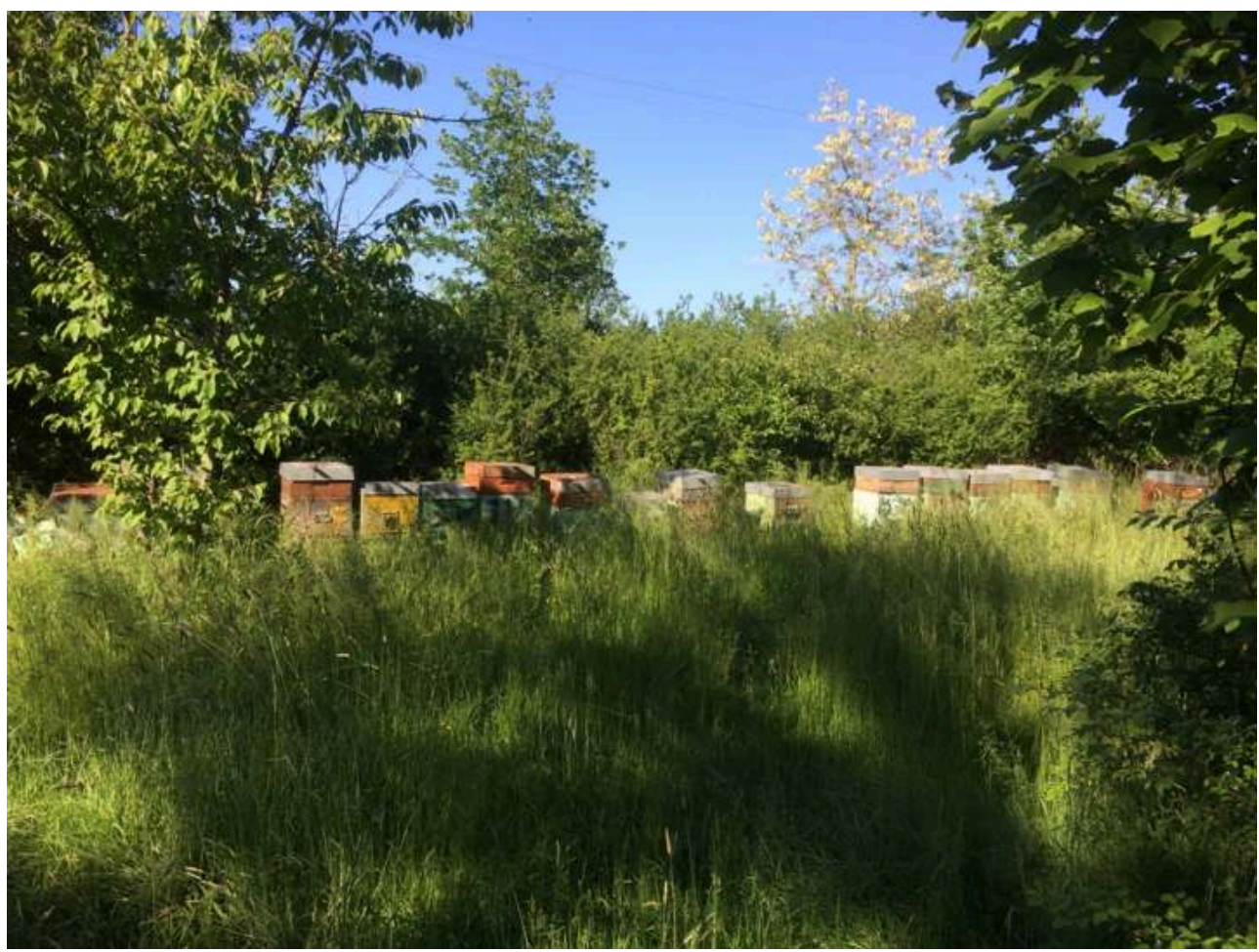

Foto 1. Ceresole d'Alba, arnie con fascia vegetale arbustiva (foto dell'autrice).

\section{NOTE}

1. A tal proposito vanno ricordati, proprio per il ruolo delle piante come sedi di conflitti istituzionali legati alle dispute sull'uso alla terra, Social Facts and Fabrication, di Sally Falk Moore (1986) e l'articolo "Agricultural Intensification and Perimetrics: Ethnoarchaeologial Evidence from Nigeria" di Glenn Stone (1994), in cui le piante da confine non assumono importanza solo 
per i benefici che apportano al suolo, ma anche perché sono il riflesso della storia del paesaggio nigeriano che l'autrice indaga.

2. Il progetto, il cui acronimo era M.A.S.K.A. (Marginal Areas: Sustainability and Know-How in the Alps), era coordinato da Laura Bonato e ha prodotto, oltre ad azioni concrete sul territorio valsusino, come un corso di tessitura e una serie di workshop per "insegnare" ai coltivatori a recuperare saperi e sementi abbandonate, anche due volumi: Aree Marginali, a cura di Laura Bonato, FrancoAngeli, Milano, 2017 e Ambientare, a cura di Lia Zola, FrancoAngeli, Milano, 2017.

3. Il mio obiettivo, allora, era comprendere il motivo per cui alcune persone scegliessero proprio le Alpi per dedicarsi a specie come la canapa, la lavanda e la segale, i cui saperi e tecniche colturali si erano persi da decenni (o non erano mai esistiti).

4. L'apicoltura nomade prevede lo spostamento delle arnie nella stagione primaverile-estiva da un'area di pianura o collina ad una di montagna; ha lo scopo di permettere alle api di sfruttare più a lungo la stagione della fioritura.

5. I progetti europei che ad oggi si sono occupati del monitoraggio degli alveari, del numero di apicoltori in ogni nazione, ma anche delle misure per contrastare la moria delle api, come ad esempio EPILOBEE (Epidemiological Study on Honey Bee Colony Losses, 2012-2014), SWARMONITOR (2012-2015), SUPER-B (2014 ad oggi), fanno tutti capo al Dipartimento di Agricoltura e Sviluppo Rurale della Commissione Europea, in particolare ai programmi NAPs (National Apiculture Programmes). Attualmente sono stati finanziati quelli relativi al biennio 2020-2022 (htpps://ec.europa.eu, consultato in data 20/06/2021). Negli ultimi decenni il declino delle popolazioni di api è stato anche causato da parassiti che mettono in seria difficoltà la sopravvivenza delle famiglie, come la varroa, un acaro che attacca sia le covate di api, sia gli individui adulti, o l'imenottero predatore vespa velutina. La questione dei parassiti esula da questo contributo, per cui non approfondirò questo tema: segnalo tuttavia uno studio condotto da Marco Porporato sugli effetti dannosi della vespa velutina (2014). Sempre Marco Porporato è a capo di un progetto di Public Engagement dell'Università di Torino per l'a.a. 2021-2022 sul monitoraggio degli impollinatori selvatici nella città metropolitana di Torino.

6. In piemontese i margari sono sia gli allevatori sia gli addetti alla gestione dei bovini durante l'alpeggio che possono anche svolgere mansioni legate alla caseificazione.

7. Le guide selvocolturali menzionate rientrano nelle azioni messe in atto dal Piano di Sviluppo Rurale della Regione Piemonte 2014-2020 e in particolare nelle Misure 10 (Agroambiente) e 11 (Agricoltura Biologica). Le due Misure attualmente sono state prorogate almeno a tutto il 2021 (www.regionepiemonte.it, consultato in data 25-06-2021).

\section{RIASSUNTI}

Il mio contributo prende in considerazione i tentativi, messi in atto da alcuni apicoltori, di "rinselvatichire" e "ripopolare" un paesaggio compromesso dall'uso estensivo di pesticidi e monocolture, attraverso la semina e la piantumazione di specie spontanee per garantire nutrimento alle loro api. Il caso di studio si inserisce in un ambito di analisi più ampio, sostenuto dalla prospettiva teorica della svolta ontologica e multispecie, che mette in risalto come specie diverse riescano a entrare in relazione tentando non solo di sopravvivere, ma anche di vivere "con". Fulcro e punti nodali di riferimento di questi intrecci relazionali sono le specie vegetali (in particolare le piante che marcano i confini) ma anche gli esseri umani e le api. 
My essay deals with the attempts acted by beekeepers, who engage in a process of introduction of spontaneous herbs and plants in order to feed their bees, of "rewildening" and "repopulating" landscapes suffering from monocropping and excessive use of pesticides. My case study relies on the ontological and multispecies approach which addresses the issue of different species' coexistence, striving not only to survive but also to live "with", in a bundle of relations. The essay explores in particular the multispecies issue taking into account border plants and their relations with humans and bees.

\section{INDICE}

Keywords : multispecies perspective, relations, human-plants relations, rewildening, bees Parole chiave : prospettive multispecie, relazionalità, uomo-piante, rinselvatichimento, api

\section{AUTORE}

\section{LIA ZOLA}

Dipartimento di Lingue e Letterature Straniere e Culture Moderne, Università di Torino lia.zola@unito.it 